Proceedings

\title{
A Systematic Review of Urban Heat Island Studies in West Africa
}

Benjamin Obe ${ }^{1, *}$ and Tobi Morakinyo ${ }^{1,}$

1 University College Dublin; benjamin.obe@ucdconnect.ie

1 University College Dublin; tobi.marakinyo@ucd.ie

* Correspondence: benjamin.obe@ucdconnect.; Tel.: (+353899644549)

\begin{abstract}
This study critically and systematically reviews the state-of-the-art on UHI research in west Africa region to identify and bridge the gap with other global literature. Focusing on 48 studies extracted through a laid down protocol, we reviewed, integrated and analysed in detail the causative factors of UHI, methods of measurement, the intensities, variabilities, impacts and mitigation strategies while critiquing the methods and limitations of the studies. Of the 16 countries in the region, UHI-related research have been conducted in only 5 countries, indicating a wide knowledge gap. Also, inconsistencies in the scale of study, data collection methods and analysis were observed, indicating that studies have not used a universal and specific protocol to define the choice of location for an urban and a rural area.
\end{abstract}

Keywords: Urban Heat Island, west Africa, Temperature, Review, systematically

\section{Introduction}

Due to its geographical location, background climate and recent large-scale urban sprawl as well as high poverty level, west Africa is most susceptible to the impact of climate change such as extreme heat [1]. A slight increase in temperature could trigger the thermal condition to be above the heat-health threshold. For example, a recent study by [2] in Kumasi, Ghana, found relatively higher Urban Heat Island Intensity(UHII) than those experience in European cities during the 2016 heatwave indicating the extent of Urban Heat Islands (UHI) in west Africa region. The problem will become more intense and frequent in the future based on scientists' projections e.g [3]-[5]. Although, the urban transition in west African cities offers tremendous social and infrastructural development opportunities. This is however made possible at the expense of the local climate. Even though human presence is part of nature, sustainable cities will be made possible if human activities respect the ecological system. The environmental equilibriums have recently been challenged with the pace of urbanization in Africa that exceeds the critical value of population density.

Despite all these, unfortunately, the understanding of the dynamics of Urban Heat Island (UHI) menace is still limited over this region [6]. This review article attempts to collate existing knowledge on the subject within west Africa. The specific goal is to 
identify critical gaps in UHI studies and the state-of-the-art methodologies and define some key research questions to accommodate future studies to achieve sustainable and resilient cities. To achieve this, we exhaustively review various studies on UHI in west Africa from 1985 to 2021 to understand the trend of existing studies with respect to the determining factors, adopted methods of measurement, the intensity and variability, impacts and mitigation strategies of UHI. The review will also tend to find topics and gaps that need more attention from researchers to inspire proactive urban climate actions in the future.

\section{Geography and climate of the study area}

2.1 Study Area

1

Figure 1. Geographical map of west Africa.

The systematic review considers UHI studies written in English over 16 countries in the region, covering a landmass of approximately 6 million $\mathrm{km}^{2}$ and an estimated population of 381 million people [7].

\subsection{Literature search and screening}

Different databases (Scopus, Google Scholar and web of science) were employed for an intensive literature search. Search terms such as Urban climate, Urban Heat Island, Heat stress, and Land surface temperature were separately combined with west African country's names. Since this systematic review focuses on Urban Heat Island over the west African domain, we limit the findings to cities within the domain and also set a subfield category using the online database. The search result returned $\mathbf{2 4 4}$ articles.

After the initial online extraction, a rigorous manual check was performed by reading the abstract and assessing whether the articles were relevant to the scope of the review. For an article to be selected, it must address the research objectives stated above; that is, it must address either of UHI development, measurements, characteristics, causes and impacts and mitigation strategies and changes in land use and surface composition in relation to Land Surface Temperature (LST. We however selected only studies written in English. Even with this broader scope, only 48 articles were selected and reviewed with case studies over 21 cities in west Africa. 


\section{Results and Discussion}

Whereas a Canopy Layer Urban Heat Island (CUHI) is based on the air temperature anomaly in the urban area, a Surface Urban Heat Island (SUHI) is based on the Land Surface Temperature (LST) anomaly in the urban areas in comparison to a rural area. A surface review of the 48 selected articles showed that the articles could broadly be classified into 3 groups defined by the type of UHI considered and focus of the study; approximately $58.3 \%$ focused on SUHI, 35.4\% considered CUHI and approximately $12.5 \%$ focused on UHI mitigation potentials with green infrastructures.

For studies in the SUHI group, there are two sub-categories: those studies that really investigated SUHI based on the traditional definition of differential LST between a urban and a defined rural reference area. Only 7 (25\%) of the 28 SUHI studies could be classified into this category. Whereas 21 studies only used LST as a proxy for UHI. They either investigated Land Use Land Cover (LULC) dynamics using remotely sensed data to establish the link between LULCC, LST, as well as variations in the intensities on a spatial and temporal scale. This shows that $75 \%$ of the SUHI studies did not intrinsically investigate the UHI effect but examined whether Spatio-temporal factors are related to LST (figure 2a). For the CUHI group, studies were analyzed based on data source and measurement approach such as the number of transect points and instrument set up with respect to the height as well as justification for urban and rural site selection. The analytical methods employed in estimating the Canopy Urban Heat Island Intensity (CUHII) were also evaluated, $64.3 \%(\mathrm{n}=9)$ of the CUHI study employed fixed method of temperature, $20 \%(n=3)$ of the study utilized the mobile traverse method, whereas $26.7 \%(n=4)$ used data from weather stations. Figure $2 b$ shows the variations of the mean maximum CUHII as reported in each study. Somehow, only $63.3 \%$ of the CUHI studies estimated the CUHII. Significant variations in the CUHII were observed in the studies. This variation may be associated with the size and scale, measurement period and analytical method of the UHI study accordingly, as there is no objective definition of urban areas, which gives rise to inconsistencies in the choice of location for placing the instrument.

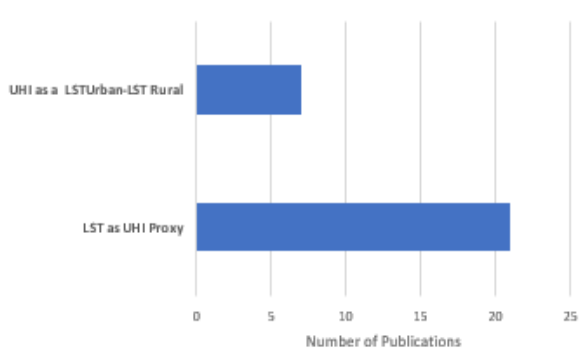

(a)

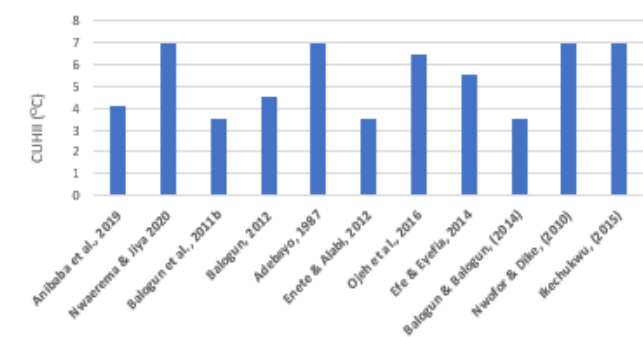

(b) 


\section{Conclusions}

The reviewed studies have generally evident the abrupt increase in urbanization and corresponding urban warming, particularly in the last two decades; A large proportion of UHI studies reviewed focused on LST changes to evident SUHI powered by the open-access Landsat data. The studies have, however, incorrectly presumed a vertical homogeneity for impervious and built up areas. However, a smaller proportion of the studies classified the urban area in terms of density variations (High density, medium density and low density). This is a step toward evaluating an intra-UHI. To improve the effectiveness of measuring the magnitude of the UHI effect in cities worldwide, a uniform and standardized method of land use classification for easy inter-city comparison, the Local Climate Zone(LCZ), is proposed over this region. This will further support the Urban Canopy Parametrisation (UCP) needs of mesoscale and microscale modeling.

Separately from inconsistencies and an awkward classification of urban and rural sites, 3 other gaps were noticed from this systematic review; i. inconsistencies in the relevant period of interest ii Inadequate cross-validation of spatial and temporal variations in UHI and iii. the lagging effect of meteorological factors was not well accounted for. Therefore, we propose the adoption of LCZ scheme with its robust UCP for a sparse data region like West Africa in UHI Modelling.

Funding: This research received no external funding.

Conflicts of Interest: The authors declare no conflict of interest.

\section{References}

1. Di Ruocco A., Gasparini P., Weets G. (2015) Urbanisation and Climate Change in Africa: Setting the Scene. In: Pauleit S. et al. (eds) Urban Vulnerability and Climate Change in Africa. Future City, vol 4. Springer, Cham. https://doi.org/10.1007/978-3-319-03982-4_1

2. I. Buo, V. Sagris, I. Burdun, and E. Uuemaa, “Estimating the expansion of urban areas and urban heat islands (UHI) in Ghana: a case study," Nat. Hazards, vol. 105, no. 2, pp. 1299-1321, Jan. 2021, doi: 10.1007/s11069-020-04355-4.

3. G. Ceccherini, S. Russo, I. Ameztoy, A. Francesco Marchese, and C. Carmona-Moreno, "Heat waves in Africa 19812015, observations and reanalysis," Nat. Hazards Earth Syst. Sci., vol. 17, no. 1, pp. 115-125, Jan. 2017, doi: 10.5194/nhess-17-115-2017.

4. P. J. Marcotullio, C. Keßler, and B. M. Fekete, “The future urban heatwave challenge in Africa: Exploratory analysis," Glob. Environ. Chang., vol. 66, Jan. 2021, doi: 10.1016/j.gloenvcha.2020.102190.

5. V. Murray and K. L. Ebi, "IPCC Special Report on Managing the Risks of Extreme Events and Disasters to Advance Climate Change Adaptation (SREX)," J. Epidemiol. Community Health, vol. 66, no. 9, pp. 759-760, 2012, doi: 10.1136/jech-2012-201045.

6. S. Chapman, J. E. M. Watson, A. Salazar, M. Thatcher, and C. A. McAlpine, “The impact of urbanization and climate change on urban temperatures: a systematic review," Landscape Ecology, vol. 32, no. 10. Springer Netherlands, pp. 1921-1935, Oct. 08, 2017, doi: 10.1007/s10980-017-0561-4.

7. UN Department of Economics and Social Affairs, “World Population Prospects - Population Division - United Nations," The International Journal of Logistics Management, 2015. https://population.un.org/wpp/ (accessed Aug. 26, 2021) 